\title{
The politics of redistributive social insurance
}

\author{
Jean Hindriks* \\ Queen Mary, University of London \\ and CORE, Belgium \\ Philippe De Donder \\ GREMAQ and IDEI, Toulouse
}

December 2000

Revised 15 October 2001

\begin{abstract}
This paper analyses the political support for a social insurance that includes elements of redistribution when there exists an imperfect private insurance alternative. Individuals differ both in their income and risk. The social insurance is compulsory and charges an income-related contribution with pooling of risks. The private insurance is voluntary and charges a contribution based on individual risks. However due to the adverse selection problem, private insurance companies provide only partial insurance. Adopting a non-expected utility model, we show that there is a general majority support for social insurance and that this support is increasing with risk aversion. We also show that a mixed insurance is politically impossible, regardless of the degree of redistribution of social insurance and the joint distribution of risk and income in the population. Lastly, we analyse how the political support for social insurance is affected by any change in its redistributive component and the possibility of using genetic tests.
\end{abstract}

Keywords:majority rule, social insurance, redistribution, adverse selection. JEL classification: $\mathrm{H} 23, \mathrm{H} 50$

* Economics department, Queen Mary,University of London, Mile End Road, London E1 4NS, England. Email:j.hindriks@qmw.ac.uk. 


\section{Introduction}

Most OECD countries rely heavily on the public sector to provide insurance. ${ }^{1}$ Since insurance is essentially a private good, we may wonder why it is so. The present paper is an attempt to answer that question. It is then a contribution to the existing literature on the public provision of private goods.

Typically, social insurance contributions are not related to risk but rather to income levels. Social insurance thus implies redistribution in two ways with mandatory participation. First, there is redistribution from low to high risks because contributions do not depend on individual's morbidity or risk: higher-risk individuals pay less than their expected risk while lowerrisk individuals pay more. Second, since contributions increase with income, higher-income individuals pay more than lower-income individuals even if they have the same risk. The overall amount of redistribution will depend on the covariance between risks and income. ${ }^{2}$

If risks are negatively related to income so that the poor face higher risks on average then we have an obvious distributive argument for public provision of social insurance. Moreover the existing literature has shown that, contrarily to the tax principle (Guesnerie, 1995), it is not possible to replicate the redistributive effect of social insurance through the income tax. ${ }^{3}$ The reason is that redistributing through social insurance from low to high risks does not involve the same distortion as redistributing directly from the high-income to the low-income.

\footnotetext{
${ }^{1}$ As for health insurance, a recent OECD report reveals that for many countries a very high proportion of health spendings were accounted for by the state (OECD Health Data 2001). According to this study in 1998 state spending as a proportion of total spending on health was $80 \%$ in Germany, $89 \%$ in France, $67 \%$ in the Netherlands, $67 \%$ in Italy, $73 \%$ in Austria, and $84 \%$ in Britain. In terms of total spendings, Germany spent $10 \%$ of GDP on health, France spent $9.5 \%$ but Britain spent just $6.8 \%$. As for pensions, Börsch-Supan et al.(2001) report that the proportion of retirement benefits coming from public insurance was on average $85 \%$ in Germany, $65 \%$ in Great-Britain, $50 \%$ in the Netherlands, $45 \%$ in the US and $42 \%$ in Switzerland.

${ }^{2}$ See Wagstaff et al. (1999) for a recent international comparison of the redistributive effect of health care systems; and Coronado et al (2000) for a study of the redistributive effect of Social Security over lifetime in the US taking into account notably difference in life expectancies.

${ }^{3}$ See Rochet(1991) and Cremer and Pestieau(1996) for the case where contributions are not related to income, and Blomqvist and Horn (1984) and Petretto (1999) for incomerelated contributions.
} 
According to this literature, the case for social insurance relies heavily on the negative association between risks and income combined with a social desire to redistribute from the rich to the poor. The natural questions are therefore whether it is possible to give a justification for social insurance when the poor do not necessarily face higher risk on average than the rich and when there is no benevolent planner to impose this redistribution from the rich to the poor. The purpose of this paper is to take up these issues by focusing instead on the political justification for social insurance given various possible relations between risks and income. We will show that, unlike the normative approach, the political demand for social insurance is stronger when risk and income are positively correlated.

For insurance to be publicly provided it must be supported by a majority. There has been some work on the political economy of the public provision of private goods, in particular health care. Usher (1977) considers public provision uniform to all individuals and financed by a proportional income tax so that given the positively skewed distribution of income a majority of individuals benefit from public provision provided that the heterogeneity in tastes for the particular good is not too high. Otherwise the redistributive gain of public provision would be offset by the cost of uniform provision. To accommodate this heterogeneity problem some authors have considered the case where public provision can be supplemented by private consumption (see Anderberg, 1999, Casamatta et al., 2000, Epple and Romano, 1996, Peterson, 1986, Pauly, 1992, and Gouveia 1997). The main feature of these papers is that the level of public provision will tend to reflect the preference of the median voter with the implication that social insurance will be organized in the interests of the middle class. In particular in Gouveia (1997) both the poor and the rich would like a lower public provision of health insurance: the poor because there is an income effect and the rich because they can get a better price with private insurance. Gouveia obtains the result that a majority will support a mixed provision of insurance. Anderberg (1999) has the same result in a model where individuals differ only in their risk and Casamatta et al. (2000) in a model where individuals differ only in their income.

In this paper we shall consider individuals differing both in their income and their risk of incurring the same damage. The model will thus describe cost insurance rather than earnings insurance. That is, the benefit of insurance is a reimbursement of the costs incurred but not a compensation for earnings loss. This assumption, while keeping the model tractable, can be justified empirically by the observation that the responsibility of providing 
earnings compensation has been partly shifted to the employer. ${ }^{4}$ We can capture to some extent this aspect of insurance against earnings loss in our model by modifying the contribution schedule of social insurance. Varying the contribution scheme from proportional income taxes to uniform taxes we can approximate the equivalence principle according to which benefits are proportional to contributions. The main distinctive feature of our model is that we assume away the income effect in the demand for insurance so that we do not get the peculiarity that the poor favour less social insurance than the middle class. We do this by adopting Yaari $(1986,1987)$ 's dual theory of choice under uncertainty (i.e., risk aversion without diminishing marginal utility of income). Beside presenting independent interest, this modelling of risk aversion will allow the derivation of a rich set of insights that could hardly be obtained with the expected utility model. We shall also consider the possibility to supplement social insurance with private insurance.

Another important difference with previous contributions is that we shall take private insurance as imperfect. It is indeed surprising that most contributions cited above either ignore the problem of adverse selection by assuming perfect private insurance or assume pooling private insurance. However insurance is plagued with adverse selection, which makes voluntary individual insurance inefficient and provides an efficiency argument for compulsory social insurance. ${ }^{5}$ Indeed, in most OECD countries social insurance is compulsory. Two major exceptions in health care are the Netherlands and the US. In these countries, a significant portion of the population was not subject to compulsory health insurance. ${ }^{6}$ Given the increase in the number of uninsured individuals, the Netherlands have adopted the Dekker reform which aims to make insurance compulsory.

Our main results are twofold. First, we demonstrate that there is in general a majority support for social insurance (regardless of the correlation between risk and income) and that this majority support for social insurance increases with risk aversion and is robust to any change to its redistributive

\footnotetext{
${ }^{4}$ In Germany, for example, employers have to pay 80-100 per cent of regular earnings during the first six weeks of sickness.

${ }^{5}$ Although in many cases the stakes are high enough to induce the insurers to find out consumers' types, this may be institutionally impossible. Indeed in some countries it is illegal to discriminate between consumers according to their genetic background. In 18 states of the US, legislation prevents insurers to deny insurance coverage or charge higher premium on genetic grounds; whereas France has imposed a moratorium of 5 years on the use of such tests (The Economist, 19 October 2000).

${ }^{6}$ In 1990, the proportion of the population covered by health social insurance was 70 per cent in the Netherlands and only 44 per cent in the US (see Besley and Gouveia, 1994, Table 5, p. 216).
} 
component. Second, we show that a system where it is possible to supplement a social insurance with voluntary private insurance is not politically feasible with the dual theory of choice under risk whatever the distribution of risk and income in the population. The reason is that due to its linearity property the dual theory tends to produce corner solutions in optimization problems, unlike the expected utility model.

The paper is organized as follows. Section 2 presents the model and the dual theory of choice under risk. Section 3 analyses social insurance and private insurance as mutually exclusive options, and derives condition for a majority to prefer social insurance against private insurance. Section 4 studies the case where social and private insurance can complement each other and shows that such a top-up system is not politically feasible. Section 5 considers how the redistributive component of social insurance affects its political support. Section 6 emphasizes the importance of adverse selection in our analysis. Section 7 offers some concluding remarks.

\section{The model}

The economy is composed of a continuum of individuals who differ in their income $w \in[\underline{w}, \bar{w}]$ (with $1 \leq \underline{w}<\bar{w}<\infty$ ) and their probability of incurring a damage $\theta \in[\underline{\theta}, \bar{\theta}]$ (with $0 \leq \underline{\theta}<\bar{\theta}<1$ ). Risk and income are distributed according to a continuous joint distribution function $H(\theta, w)$. All individuals face the same damage $d=1$ but with different probabilities.

There is no moral hazard since individuals cannot affect their probability of accident which is fixed. ${ }^{7}$ However adverse selection is introduced by assuming that each individual knows her own risk (i.e. value of $\theta$ ) which is private information. The insurance companies only know the marginal distribution of risks in the population, which is denoted by $F(\theta)$. So $F(\theta)$ is the fraction of the population with probability of accident less or equal to $\theta$. The mean risk is $\theta_{\mu}=\int_{\theta}^{\bar{\theta}} \theta d F(\theta)$ and the median risk is $\theta_{m}=F^{-1}(1 / 2)$. We assume that the government also knows the mean risk $\theta_{\mu}$ and can observe income levels which are distributed according to the marginal distribution function $G(w)$. The mean income is $w_{\mu}=\int_{w}^{\bar{w}} w d G(w)$ and the median income is $w_{m}=G^{-1}(1 / 2)$. Any insurance contract consists of a premium $\pi$ and a coverage rate $\delta \in[0,1]$ which is the proportion of the damage reimbursed.

\footnotetext{
${ }^{7}$ Moral hazard is assumed away because it is believed that it would affect private and public insurance in the same way, and so it should not influence the choice between the two systems.
} 
We model individuals' risk preferences using Yaari (1987)'s dual theory (DT). We first give a general description of this approach before applying it to our model. Let wealth $X$ be a random variable distributed over $[\underline{x}, \bar{x}]$ according to the distribution function $\Psi(x)$. Yaari's representation of preferences is dual to the expected utility theory (EU) in the sense that it is linear in wealth but non linear in probabilities. Probabilities are transformed by a function $\phi$ defined on the distribution function $\Psi(x) .{ }^{8}$ More precisely, DT preferences over $X$ are given by

$$
V(X)=\int x \phi^{\prime}(\Psi(x)) d \Psi(x)
$$

where $\phi(0)=0, \phi(1)=1$ and $\phi^{\prime}()>.0 . \quad \phi^{\prime}($.$) are non-negative weights$ adding up to one. Attitude towards risk is conveyed entirely by the shape of $\phi($.$) . Risk aversion is characterized by the concavity of \phi($.$) , i.e. \phi^{\prime \prime}()<.0 .^{9}$ In this case, bad outcomes (with low $\Psi(X)$ ) receive higher weights than good outcomes (with high $\Psi(X)$ ). In other words, $V(X)$ is the certaintyequivalent of $X$ computed as a weighted average of outcomes in which bad outcomes are given high weight while good outcomes are given low weight. Since $V(X)$ is linear in wealth, this approach separates attitude towards risk from attitude towards wealth and as we shall see shortly it allows the derivation of a rich set of insights. We also know from Machina (1995) that most (though not all) of the standard results in insurance theory are robust to this departure from the expected utility hypothesis. There is however a key difference related to the order of risk aversion. ${ }^{10}$ When risk aversion is of order 1 as in the DT, it could be optimal for a policyholder to buy full insurance even above the fair price. This is because he derives positive benefits from the last dollar of coverage. By contrast under EU, risk aversion is of the second order and the benefit from the last dollar of coverage is zero. Therefore nobody would find profitable to buy complete insurance slightly above fair price in the EU model eventhough this is common practice. ${ }^{11}$

\footnotetext{
${ }^{8}$ Alternatively this probability transformation function could be defined on the decumulative distribution function $1-\Psi$ such as in Yaari (1987).

${ }^{9}$ If the probability transformation function were defined on the decumulative distribution function, then risk aversion would be characterised by the convexity of $\phi(1-\Psi)$.

${ }^{10}$ For the definition of the order of risk aversion, see Segal and Spivak (1990). An excellent survey of the various definitions of risk aversion is provided in Cohen (1995).

${ }^{11}$ Mossin (1968) was the first to point out this anomaly of the EU model. Note that in our model with a fixed damage $(d=1)$, partial coverage is equivalent to a deductible.
} 
Another distinctive property of insurance under DT is that insurance cannot be a Giffen good as in the EU model. ${ }^{12}$

We now apply DT to our simple two-state setting. For an individual with income $w$ facing a damage $d=1$ with probability $\theta$, insurance contract with premium $\pi>0$ and coverage rate $\delta \in[0,1]$ yields the random variable $X=(w-\pi-(1-\delta), \theta ; w-\pi, 1-\theta)$. We thus define the utility associated to this insurance contract as

$$
\begin{aligned}
V(\pi, \delta ; \theta, w) & =\phi(\theta)(w-\pi-(1-\delta))+(1-\phi(\theta))(w-\pi) \\
& =w-\pi-\phi(\theta)(1-\delta)
\end{aligned}
$$

where risk aversion is represented by $\phi(\theta)>\theta$ (and $1-\phi(\theta)<1-\theta$ ). In this paper, we further assume that $\phi(\theta)=(1+\alpha) \theta$, with $0 \leq \alpha \leq \frac{1-\bar{\theta}}{\bar{\theta}}$ (the upper bound guaranteeing that $\phi(\theta) \leq 1 \forall \theta)$. Making $\alpha$ independent of $w$ accords with our desire to disentangle risk aversion from income and will greatly simplify the analysis. Using this formulation, type $(w, \theta)$ individual's utility function from insurance contract $(\pi, \delta)$ is

$$
V(\pi, \delta ; \theta, w)=w-\pi-(1+\alpha)(1-\delta) \theta
$$

and the reservation premium $\pi=\pi(\theta)$ solves

$$
\begin{aligned}
V(\pi, \delta ; \theta, w) & =V(0,0 ; \theta, w) \\
w-\pi-(1+\alpha)(1-\delta) \theta & =w-(1+\alpha) \theta
\end{aligned}
$$

so that

$$
\pi(\theta)=(1+\alpha) \theta \delta
$$

Therefore the reservation premium exceeds the expected payout from the insurance company by a multiplicative factor $\alpha$. This indicates that risk aversion in our model takes the form of a relative markup (i.e., the ratio between the price markup and the fair price): $\alpha=(\pi(\theta)-\theta \delta) / \theta \delta$.

\section{Social insurance vs private insurance}

In this section, private and social insurance are mutually exclusive. The mandatory social insurance policy offers full coverage $\delta^{u}=1$ with a premium

\footnotetext{
${ }^{12}$ Doherty and Eeckhoudt (1995) derive this result and other properties of optimal insurance in the case of Yaari's dual model.
} 
$\pi^{u}(w)$ proportional to income but not related to individual risks. ${ }^{13}$ To break even the average premium must be equal to the average expected payout, $\int_{w} \pi^{u}(w) d G(w)=\theta_{\mu}$ with individual premium $\pi^{u}(w)=\frac{w}{w_{\mu}} \theta_{\mu}$. We then obtain the following payoff from social insurance for an agent with risk $\theta$ and income $w$ :

$$
V\left(\delta^{u}, \pi^{u}(w) ; \theta, w\right)=w-\left(\frac{w}{w_{\mu}}\right) \theta_{\mu} .
$$

Alternatively, voluntary private insurance may be provided by insurance companies. They offer a set of separating zero-profit contracts $\{\delta(\theta), \pi(\theta)\}_{\theta \in[\theta, \bar{\theta}]}$, where $\delta(\theta) \in[0,1]$ is the coverage rate and $\pi(\theta)$ is the premium for a consumer with risk $\theta$. In a fully separating equilibrium, each type $\theta \in[0,1]$ then selects the policy intended for her and each contract will break even. This requires to satisfy the following set of self-selection constraints: For all $\theta$ and $\hat{\theta}$ with $\hat{\theta} \neq \theta$,

$$
\begin{aligned}
V(\delta(\theta), \pi(\theta) ; \theta, w) & =w-\pi(\theta)-(1+\alpha)(1-\delta(\theta)) \theta \\
& \geq V(\delta(\hat{\theta}), \pi(\hat{\theta}) ; \theta, w)=w-\pi(\hat{\theta})-(1+\alpha)(1-\delta(\hat{\theta})) \theta
\end{aligned}
$$

Using the zero-profit condition (due to perfect competition among risk neutral insurance companies) every agent with risk $\theta$ is facing actuarially fair premium: $\pi(\theta)=\delta(\theta) \theta$. Incorporating this into the self-selection constraints, we obtain for all $\hat{\theta} \neq \theta$

$$
\begin{aligned}
V(\delta(\theta), \pi(\theta) ; \theta, w) & =w-\delta(\theta) \theta-(1+\alpha)(1-\delta(\theta)) \theta \\
& \geq V(\delta(\hat{\theta}), \pi(\hat{\theta}) ; \theta, w)=w-\delta(\hat{\theta}) \hat{\theta}-(1+\alpha)(1-\delta(\hat{\theta})) \theta
\end{aligned}
$$

We follow the incentive compatibility approach of Mailath (1987). A necessary condition for a separating equilibrium is that the following local incentive compatibility conditions hold: for all $\theta \in[\underline{\theta}, \bar{\theta}]$

$$
\begin{aligned}
{\left[\frac{\partial V(\delta(\hat{\theta}), \pi(\hat{\theta}) ; \theta, w)}{\partial \hat{\theta}}\right]_{\hat{\theta} \rightarrow \theta} } & =-\delta^{\prime}(\theta) \theta-\delta(\theta)+(1+\alpha) \delta^{\prime}(\theta) \theta=0 \\
& =\delta^{\prime}(\theta)[(1+\alpha) \theta-\theta]-\delta(\theta)=0 .
\end{aligned}
$$

\footnotetext{
${ }^{13}$ The assumption of proportional taxation is consistent with the way social insurance is financed in many countries through payroll taxes. We do however consider more general form of financing in Section 5.
} 
In other words, any separating equilibrium requires that no type be able to obtain a strictly higher payoff by announcing another arbitrary close type. ¿From Mailath (1987), we see that the payoff functions are such that these local necessary conditions are also sufficient. Hence the equilibrium coverage rate function $\delta(\theta)$ is the solution to the following differential equation,

$$
\delta^{\prime}(\theta)=\frac{\delta(\theta)}{\alpha \theta} \quad \forall \theta \in[\underline{\theta}, \bar{\theta}]
$$

Solving this equation and using the terminal condition $\delta(\bar{\theta})=1$ for efficiency, we get the equilibrium coverage function

$$
\delta^{*}(\theta)=(\theta / \bar{\theta})^{1 / \alpha}
$$

with the corresponding equilibrium premium function,

$$
\pi^{*}(\theta)=\delta^{*}(\theta) \theta=\theta(\theta / \bar{\theta})^{1 / \alpha}
$$

The fact that $(\theta / \bar{\theta})^{1 / \alpha}<1$ for all $\theta<\bar{\theta}$ reflects the inherent incomplete coverage resulting from the adverse selection problem on the private insurance market. The policy menu $\left\{\delta^{*}(\theta), \pi^{*}(\theta)\right\}_{\theta \in[0,1]}$ characterises the unique efficient separating zero-profit equilibrium in this continuous-type insurance model. ${ }^{14}$ Plugging the equilibrium separating contracts into the payoff functions we get

$$
\begin{aligned}
V\left(\delta^{*}(\theta), \pi^{*}(\theta) ; \theta, w\right) & =w-\pi^{*}(\theta)-(1+\alpha)\left(1-\delta^{*}(\theta)\right) \theta \\
& =w-(1+\alpha) \theta+\alpha \theta(\theta / \bar{\theta})^{1 / \alpha}
\end{aligned}
$$

\footnotetext{
${ }^{14}$ This separating equilibrium may not be a Nash equilibrium (between insurance companies). A sufficient condition for existence of a Nash equilibrium is that the marginal screening cost (trading lower coverage for lower premium), for different types must decline rapidly enough as type quality increases (Riley, 1985). When probabilities of accident are small, this condition is likely to be met (Riley, 1985). An alternative approach to the problem of non-existence of a Nash equilibrium is to introduce an additional stability property about the reactions to defections, leading to the so-called Reactive equilibrium (Riley, 1979). In Engers and Fernandez (1987) it is shown that, under the assumptions of our model, the set of efficient, zero-profit, separating contracts is the unique Reactive equilibrium. That is, it is possible to show that, for any new contract offered by a defecting insurance company, there is a corresponding reaction contract that yields profit for the reacting firm and produces losses for the defecting one.
} 
Comparing (4) and (3) reveals that for each $\theta \in[\underline{\theta}, \bar{\theta}]$ there exists a critical income level $w=w^{\circ}\left(\theta, \alpha, \theta_{\mu}, \bar{\theta}, w_{\mu}\right)$ such that individual with risk $\theta$ and income $w^{\circ}(\theta, \cdot)$ is indifferent between social insurance and private insurance. Given $\alpha, \theta_{\mu}$ and $w_{\mu}$, for each $\theta \in[\underline{\theta}, \bar{\theta}], w=w^{\circ}(\theta ; \cdot)$ solves

$$
w-(1+\alpha) \theta+\alpha \theta(\theta / \bar{\theta})^{1 / \alpha}=w-\left(\frac{w}{w_{\mu}}\right) \theta_{\mu}
$$

or

$$
\frac{w^{\circ}(\theta ; \cdot)}{w_{\mu}}=(1+\varphi(\alpha, \theta)) \frac{\theta}{\theta_{\mu}}
$$

where $\varphi(\alpha, \theta) \equiv \alpha\left(1-(\theta / \bar{\theta})^{1 / \alpha}\right) \geq 0$ is the efficiency gain from social insurance as measured by the extra coverage $1-(\theta / \bar{\theta})^{1 / \alpha}$ evaluated according to the degree of risk aversion $\alpha$. Mandatory social insurance deals better with adverse selection than voluntary private insurance and can provide better coverage. However social insurance also operates redistribution both from low risk to high risk and from high income to low income. Clearly all those individuals with above average risk and below average income will prefer social insurance both for efficiency and redistributive reasons. Some of those below average risk may also prefer social insurance if their income is sufficiently below the average so that income redistribution dominates risk redistribution. Indeed for any risk $\theta$ all individuals with income $w \leq w^{\circ}(\theta ; \cdot)$ would prefer social insurance over private insurance whereas all those with $w>w^{\circ}(\theta ; \cdot)$ would prefer private insurance. The political support for social insurance is then given by

$$
S=\left\{(\theta, w): \underline{\theta} \leq \theta \leq \bar{\theta} \text { and } \underline{w} \leq w \leq w^{\circ}(\theta ; \cdot)\right\}
$$

Note from (5) that some individuals slightly richer than the average $(w>$ $\left.w_{\mu}\right)$ and below the average risk $\left(\theta<\theta_{\mu}\right)$ may still prefer social insurance. This is because of the efficiency gain of social insurance. The indifferent voters correspond to the set of types $(\theta, w)$ satisfying equation (5). This equation defines the switching curve between private and social insurance in the $(\theta, w)$-space. Simple inspection reveals that $w^{\circ}(0 ; \cdot)=0$ and that for $\alpha>0, w^{\circ}(\theta ; \cdot)>w_{\mu}$ for all $\theta \geq \theta_{\mu}$.

Increasing the risk $\theta$ affects individual preference for social insurance in two opposite ways. First, it makes the pooling of risks more appealing. Second, it decreases the efficiency gain from social insurance as measured by $\varphi(\alpha, \theta)$, since higher risks benefit from better private coverage. However it 
is easily verified that the first effect always dominates except for the highest risks. Formally,

$$
\partial w^{\circ} / \partial \theta\left\{\begin{array}{l}
\geq \\
<
\end{array}\right\} 0 \quad \text { for } \quad \theta\left\{\begin{array}{l}
\leq \\
>
\end{array}\right\} \bar{\theta}^{1+\alpha},
$$

The position of the switching curve also depends on risk aversion $\alpha$, mean income $w_{\mu}$, and mean risk $\theta_{\mu}$. Indeed it appears immediately from equation (5) that for all $\theta, w^{\circ}(\theta ; \cdot)$ is increasing in $w_{\mu}$ and decreasing in $\theta_{\mu}$ and that $w^{\circ}(\theta ; \cdot)$ is unaffected by mean preserving changes in the joint distribution $H(\theta, w)$. It can also be verified that for each $\theta, w^{\circ}(\theta ; \cdot)$ is increasing in $\alpha .^{15}$

As an illustration, Figure 1 reports the relative critical wage, $w^{\circ} / w_{\mu}$, as a function of the relative risk, $\theta / \theta_{\mu}$, for various degrees of risk aversion (and $\theta_{\mu}=1 / 3, w_{\mu}=2$ and $\left.\bar{\theta}=2 / 3\right)$.

\section{[Insert Figure 1]}

It is now straightforward to obtain the condition on the distribution of types and risk aversion such that a majority of individuals favour social insurance.

Proposition 1 There is a majority support for social insurance against private insurance if and only if

$$
\int_{\underline{\theta}}^{\bar{\theta}} \int_{\underline{w}}^{w^{\circ}(\theta ; \cdot)} d H(\theta, w) \geq 1 / 2
$$

where $w^{\circ}(\theta ; \cdot)$ is increasing in $\theta$ for all $\theta \leq \bar{\theta}^{1+\alpha}$ and where risk aversion $(\alpha>0)$ implies that $w^{\circ}(\theta ; \cdot)>w_{\mu}$ for all $\theta \geq \theta_{\mu}$. Moreover for each $\theta \in[\underline{\theta}, \bar{\theta}]$ the upper bound $w^{\circ}(\theta ; \cdot)$ is increasing in $\alpha$ so that more risk aversion increases the political support for social insurance.

It is extremely difficult to obtain explicit conditions on the joint distribution of types such that a majority prefers social insurance. However there is one special case in which strong results are obtainable without simulation. Suppose that risk and income are independently distributed according

\footnotetext{
${ }^{15}$ From equation $(5), \operatorname{sign}\left\{\partial w^{\circ} / \partial \alpha\right\}=\operatorname{sign}\left\{1+(\theta / \bar{\theta})^{1 / \alpha}\left(\frac{\log \theta / \bar{\theta}}{\alpha}-1\right)\right\}>0$. This last inequality requires $\log (\theta / \bar{\theta})^{1 / \alpha}+\frac{1}{(\theta / \bar{\theta})^{1 / \alpha}}>1$ which holds for all $\theta$ since the left hand side decreases with $\theta$ and converges to 1 when $\theta$ tends towards $\bar{\theta}$.
} 
to symmetric marginal distributions, $F(\theta)$ and $G(w)$. Suppose also for a moment that there is no risk aversion. Setting $\alpha=0$, the indifference condition becomes $\frac{w^{\circ}(\theta ; \cdot)}{w_{\mu}}=\frac{\theta}{\theta_{\mu}}$ and all those with $\frac{w}{w_{\mu}} \leq \frac{\theta}{\theta_{\mu}}$ prefer social insurance whereas all those with $\frac{w}{w_{\mu}}>\frac{\theta}{\theta_{\mu}}$ prefer private insurance. By the symmetry of the marginal distributions and the absence of correlation between income and risk we have $\operatorname{Pr}\left(\theta, w: \frac{w}{w_{\mu}} \leq \frac{\theta}{\theta_{\mu}}\right)=\operatorname{Pr}\left(\theta, w: \frac{w}{w_{\mu}}>\frac{\theta}{\theta_{\mu}}\right)=1 / 2$. But from Proposition 1 the support for social insurance increases with risk aversion, therefore we have that strictly more than one half of the population will prefer social insurance when $\alpha>0$. To sum up:

Proposition 2 Suppose that risk and income are uncorrelated and symmetrically distributed, then for any $\alpha>0$ there is a strict majority support for social insurance against private insurance.

Keeping the assumption that $\theta$ and $w$ are independently distributed we now relax the symmetry hypothesis. We first assume that the income distribution is positively skewed but we maintain the symmetry of the risk distribution. We expect as a result of this asymmetry a greater political support for social insurance because more individuals would then benefit from the income redistribution involved in social insurance. This is confirmed by Table 1, which shows the political support for social insurance when risks are symmetrically distributed and income levels are drawn from a $\operatorname{Bet} a(a, b)$ distribution. If $a>1$ and $b>1$ the distribution is unimodal; if $\alpha<1$ and $\beta<1$ it is $\mathrm{U}$-shaped, and if $\alpha=\beta=1$ it is the uniform distribution. The degree of skewness increases with the difference $|a-b|$. The distribution is symmetric if $a=b$, positively skewed if $a<b$ and negatively skewed if $a>b$.

Table 1: Support for social insurance with uncorrelated risk and income (symmetric risk distribution and positively skewed income distribution $)^{16}$

\begin{tabular}{|l|l|l|l|l|l|}
\hline $\mathbf{b}$ & $w_{m} / w_{\mu}$ & $\boldsymbol{\alpha = 0}$ & $\boldsymbol{\alpha}=\mathbf{0 . 1}$ & $\boldsymbol{\alpha}=\mathbf{0 . 2}$ & $\boldsymbol{\alpha}=\mathbf{1} / \mathbf{3}$ \\
\hline $\mathbf{2}$ & 1 & $50.00 \%$ & $55.80 \%$ & $60.41 \%$ & $65.73 \%$ \\
\hline $\mathbf{3}$ & 0.981 & $50.27 \%$ & $56.23 \%$ & $61.52 \%$ & $66.20 \%$ \\
\hline $\mathbf{5}$ & 0.970 & $50.63 \%$ & $57.02 \%$ & $62.23 \%$ & $66.90 \%$ \\
\hline $\mathbf{1 0}$ & 0.963 & $50.83 \%$ & $57.47 \%$ & $62.27 \%$ & $67.20 \%$ \\
\hline
\end{tabular}

Continuing with uncorrelated income and risk we could expect a greater support for social insurance with a negatively skewed distribution of risk

${ }^{16} w=3 x+1$ with $x \sim \operatorname{Beta}(2, b)$ so that $w \in[1,4]$

$\theta=2 y / 3$ with $y \sim \operatorname{Beta}(2,2)$ so that $\theta \in[0,2 / 3]$ 
since this increases the number of individuals above the mean risk who then benefit from the redistribution across risks. This is indeed verified by the results presented in Table 2 where increasing the parameter $a$ increases the negative skewness of the distribution of risks and raises the political support of social insurance. This Table also shows how positive skewness of income distribution and negative skewness of risk distribution reinforce each other in favouring social insurance.

Table 2: Support for social insurance with uncorrelated risk and income (negatively skewed distribution of risk and positively skewed distribution of income $)^{17}$

\begin{tabular}{|l|l|l|l|l|l|}
\hline $\mathbf{a}$ & $\theta_{m} / \theta_{\mu}$ & $\boldsymbol{\alpha}=\mathbf{0}$ & $\boldsymbol{\alpha}=\mathbf{0 . 1}$ & $\boldsymbol{\alpha}=\mathbf{0 . 2}$ & $\boldsymbol{\alpha}=\mathbf{1} / \mathbf{3}$ \\
\hline $\mathbf{2}$ & 1 & $50.27 \%$ & $56.23 \%$ & $61.52 \%$ & $66.20 \%$ \\
\hline $\mathbf{3}$ & 1.023 & $51.08 \%$ & $59.13 \%$ & $65.00 \%$ & $69.97 \%$ \\
\hline $\mathbf{5}$ & 1.031 & $52.12 \%$ & $61.58 \%$ & $66.78 \%$ & $70.85 \%$ \\
\hline $\mathbf{1 0}$ & 1.033 & $52.28 \%$ & $62.32 \%$ & $66.88 \%$ & $71.08 \%$ \\
\hline
\end{tabular}

We now study the effect of introducing some correlation between income and risk. To do this, we compute the proportion of individuals favouring social insurance when risks are symmetrically distributed and income distribution is skewed to the right according to parameter $b$. For every degree of skewness $b \in\{2,3,5,10\}$, we report in Table 3 the political support for social insurance for each degree of correlation $\operatorname{corr}(w, \theta) \in\{-0.99,0,+0.99\}$.

The result we obtain is that, irrespective of the skewness of the income distribution (i.e. the value of $b$ ), increasing the correlation between risk and income increases the political support for social insurance in the presence of risk aversion. Moreover the effect of correlation is accentuated by risk aversion. Indeed without risk aversion the correlation of income and risk has little effect on the support for social insurance. To understand why positive correlation is more favourable to social insurance, consider Figure 1 which depicts $w^{\circ} / w_{\mu}$ as a function of $\theta / \theta_{\mu}$ for different values of $\alpha$. When $\alpha=0$, the switching line corresponds to the $45^{\circ}$ line, $w^{\circ} / w_{\mu}=\theta / \theta_{\mu}$ and the correlation between $w$ and $\theta$ does not affect much the probability masses on both sides of this line. Positive correlation has only the effect of tightening the link between benefits and contributions. But when $\alpha$ increases, the switching line bulges northward, inducing some individuals just above the $45^{\circ}$ line to switch their support in favour of social insurance. Since positive correlation between $w$ and $\theta$ results in a higher proportion of individuals

\footnotetext{
${ }^{17} w=3 x+1$ with $x \sim \operatorname{Beta}(2,3)$ so that $w \in[1,4]$
}

$\theta=2 y / 3$ with $y \sim \operatorname{Beta}(a, 2)$ so that $\theta \in[0,2 / 3]$ 
distributed around the $45^{\circ}$ line compared to negative correlation, it follows that the political support for social insurance is higher when income and risk are positively correlated.

Table 3: Support for social insurance with correlated income and risk (symmetric distribution of risk and positively skewed distribution of income $)^{18}$

\begin{tabular}{|c|c|c|c|c|c|}
\hline b & $\operatorname{corr}(w, \theta)$ & $\boldsymbol{\alpha}=\mathbf{0}$ & $\alpha=0.1$ & $\alpha=0.2$ & $\alpha=1 / 3$ \\
\hline 2 & -0.99 & $50.05 \%$ & $54.37 \%$ & $58.08 \%$ & $62.17 \%$ \\
\hline 2 & 0 & $50.00 \%$ & $55.80 \%$ & $60.41 \%$ & $65.73 \%$ \\
\hline 2 & 0.99 & $50.37 \%$ & $64.18 \%$ & $74.08 \%$ & $80.57 \%$ \\
\hline 3 & -0.99 & $50.90 \%$ & $55.33 \%$ & $59.30 \%$ & $62.97 \%$ \\
\hline 3 & 0 & $50.27 \%$ & $56.23 \%$ & $61.52 \%$ & $66.20 \%$ \\
\hline 3 & 0.99 & $53.00 \%$ & $65.90 \%$ & $73.88 \%$ & $81.43 \%$ \\
\hline 5 & -0.99 & $51.42 \%$ & $56.00 \%$ & $60.02 \%$ & $63.92 \%$ \\
\hline 5 & 0 & $50.63 \%$ & $57.02 \%$ & $62.23 \%$ & $66.90 \%$ \\
\hline 5 & 0.99 & $54.00 \%$ & $66.33 \%$ & $73.65 \%$ & $80.08 \%$ \\
\hline 10 & -0.99 & $51.95 \%$ & $56.82 \%$ & $61.08 \%$ & $65.02 \%$ \\
\hline 10 & 0 & $50.83 \%$ & $57.47 \%$ & $62.27 \%$ & $67.20 \%$ \\
\hline 10 & 0.99 & $54.48 \%$ & $63.72 \%$ & $70.73 \%$ & $76.15 \%$ \\
\hline
\end{tabular}

\section{Impossibility of top-up system}

In this section we introduce the possibility of supplementing social insurance with voluntary private insurance. We show that all individuals either prefer full social insurance or no social insurance at all. In other words, no one will vote for a partial social insurance complemented by voluntary private insurance.

Let $\delta^{u} \in[0,1]$ and $\pi^{u}(w)=\delta^{u} \frac{w}{w_{\mu}} \theta_{\mu}$ be the redistributive social insurance policy. Individuals can freely supplement this public insurance with a private insurance leading to a total coverage for type $\theta$ equal to $\delta(\theta)$ where the extra private coverage $\delta(\theta)-\delta^{u}$ is purchased at a fair price $\left(\delta(\theta)-\delta^{u}\right) \theta$ and satisfies the incentive compatibility constraints. The corresponding payoff function for an individual with risk $\theta$ and income $w$ is,

$$
V\left(\delta^{u}, \pi^{u}(w), \delta(\theta), \pi(\theta) ; \theta, w\right)=w-\delta^{u} \frac{w}{w_{\mu}} \theta_{\mu}-\left(\delta(\theta)-\delta^{u}\right) \theta
$$

\footnotetext{
${ }^{18} w=3 x+1$ with $x \sim \operatorname{Beta}(2, b)$ so that $w \in[1,4]$

$\theta=2 y / 3$ with $y \sim \operatorname{Beta}(2,2)$ so that $\theta \in[0,2 / 3]$
} 


$$
-(1+\alpha)(1-\delta(\theta)) \theta
$$

In any separating equilibrium, the following necessary local incentive compatibility condition must hold: for all $\theta \in[\underline{\theta}, \bar{\theta}]$

$$
\begin{aligned}
{\left[\frac{\partial V\left(\delta^{u}, \pi^{u}(w), \delta(\hat{\theta}), \pi(\hat{\theta}) ; \theta, w\right)}{\partial \hat{\theta}}\right]_{\hat{\theta} \rightarrow \theta} } & =-\left(\delta(\theta)-\delta^{u}\right)-\delta^{\prime}(\theta) \theta+(1+\alpha) \delta^{\prime}(\theta) \theta \\
& =\alpha \theta \delta^{\prime}(\theta)-\left(\delta(\theta)-\delta^{u}\right)=0
\end{aligned}
$$

From Mailath(1987), it is easily seen that the payoff functions are such that the local incentive compatibility condition is also sufficient, implying that the solution to the equation above defines the separating equilibrium for the continuous-type case. Hence the separating equilibrium coverage rate function $\delta(\theta)$ solves the following differential equation,

$$
\delta^{\prime}(\theta)=\frac{\delta(\theta)}{\alpha \theta}-\frac{\delta^{u}}{\alpha \theta} \quad \forall \theta \in[\underline{\theta}, \bar{\theta}]
$$

Setting $\delta(\bar{\theta})=1$ for efficiency, the unique solution is,

$$
\delta^{*}(\theta)=\left(1-\delta^{u}\right)(\theta / \bar{\theta})^{1 / \alpha}+\delta^{u} .
$$

In particular, setting $\delta^{u}=0$ we get the same expression for $\delta^{*}(\theta)$ as in the previous section. Notice that in equilibrium, for all types total coverage is increasing with social insurance coverage $\delta^{u}$ since $(\theta / \bar{\theta})^{1 / \alpha}<1$ for all $\theta<\bar{\theta}$. Hence private insurance decisions are affected by the level of social insurance coverage but there is no complete crowding out of private insurance by social insurance. This is because social insurance cross-subsidises high risks and thereby enables private insurance to offer better coverage to low risks without violating the incentive constraints, making possibly both high risks and low risks individuals better off. ${ }^{19}$ We shall see shortly that this complementarity between private and public insurance is not enough to favour a mixed system.

Inserting this equilibrium policy into the payoff function we get

$$
V\left(\delta^{u}, \pi^{u}(w), \delta^{*}(\theta), \pi^{*}(\theta) ; \theta, w\right)=w-\delta^{u} \frac{w}{w_{\mu}} \theta_{\mu}-\left(\delta^{*}(\theta)-\delta^{u}\right) \theta
$$

\footnotetext{
${ }^{19}$ See Eckstein et al., 1985, for a similar result with VNM preferences.
} 


$$
\begin{aligned}
& -(1+\alpha)\left(1-\delta^{*}(\theta)\right) \theta \\
= & w-\delta^{u} \frac{w}{w_{\mu}} \theta_{\mu}-\left(1-\delta^{u}\right)(\theta / \bar{\theta})^{1 / \alpha} \theta \\
& -(1+\alpha)\left(1-\delta^{u}-\left(1-\delta^{u}\right)(\theta / \bar{\theta})^{1 / \alpha}\right) \theta
\end{aligned}
$$

Differentiating the above payoff functions with respect to $\delta^{u}$ we get

$$
\frac{\partial V(\cdot)}{\partial \delta^{u}}=\left(\theta-\frac{w}{w_{\mu}} \theta_{\mu}\right)+\alpha \theta\left[1-(\theta / \bar{\theta})^{1 / \alpha}\right] .
$$

This expression being independent of $\delta^{u}$, individuals have corner preferences with respect to $\delta^{u}$. Moreover it is easily seen that for any $\theta \in[\underline{\theta}, \bar{\theta}]$, there exists an agent with a critical income $w=w^{\circ}\left(\theta, \alpha, \theta_{\mu}, \bar{\theta}, w_{\mu}\right)$ who is indifferent to any change in the public coverage rate $\delta^{u}$. Notice that this marginal type is the same as in the previous section where social insurance coverage was set equal to one, see equation(5). This equivalence is due to the fact that the preference for social insurance is independent of the level of public coverage. Finally, notice that for each $\theta$ all those with income $w<w^{\circ}(\theta ; \cdot)$ prefer full public coverage and all those with income $w \geq w^{\circ}(\theta ; \cdot)$ prefer zero public coverage. Therefore depending on the probability mass of each group, a majority prefers either no social insurance at all, or full social insurance. In this sense, there is a complete political resistance to a mixed system under the dual theory, unless there are some other incentive considerations preventing full public coverage (e.g., moral hazard and distortionary effect of social insurance) or no social insurance at all (e.g., universal access to basic insurance).

We are now in a position to state the following Proposition.

Proposition 3 Suppose that agents can supplement social insurance with private insurance and that they vote on the degree of social insurance coverage. Then under the dual theory of risk, for any joint distribution of risk and income in the population, a majority would favour either full social insurance or no social insurance at all. Therefore mixed insurance is not politically sustainable unless complete public coverage is precluded for some incentive reasons.

This result stands in sharp contrast to previous work showing that there is always a majority in favour of a mixed insurance even if full public coverage is possible. The reason of the difference lies in our adopting the dual theory 
of risk. In Yaari's dual model, where risk aversion is of the first order, it is optimal to buy full insurance even if the premium is slightly above the fair price. This is because the agents derive positive benefit from the last unit of coverage. In contrast under the expected utility model, where risk aversion is of the second order, the last unit of coverage has zero benefit and thus nobody will buy full insurance above the fair price. This means that in most cases the majority will only demand partial social insurance which high risk individuals will find profitable to top up with some private insurance.

It would be a misinterpretation of Proposition 3 to dismiss the dual theory on the grounds that mixed insurance exists in the real world. The result that there cannot be a majority support for a mixed system is not to be taken as a general feature of the dual theory of risk aversion. For instance, introducing distortionary financing of social insurance would make additional social insurance coverage increasingly costly and could lead a majority of voters to support mixed insurance. The main point of proposition 3 is rather that the previous work, using the expected utility, may have overestimated the political demand for mixed insurance. Our result thus suggests that the political demand for a mixed system relies heavily on the modelling of choice under uncertainty.

It is also important to note that the case for a political support for mixed insurance made in previous work such as Anderberg (1999) and Gouveia (1997) rests also on the assumption of pooling insurance on the private market. With pooling private insurance, public provision is less appealing as it adversely affects the price of private insurance by crowding out the good risks from the market. This is not the case in our model, where the price of fully separating private contracts is not affected by social insurance. The assumption of uniform pricing of private insurance is difficult to defend because individuals actually reveal their type by purchasing different amounts of insurance, so that insurance companies could gain by exploiting this information. This constitutes a second reason why the previous literature may have overestimated the political support for mixed insurance.

\section{Effects of changing the degree of redistribution}

In this section we extend our analysis to allow for different degrees of redistribution of social insurance. To do so we suppose that the social insurance 
premium for a coverage rate $\delta^{u} \in[0,1]$ is

$$
\pi^{u}(w ; \gamma)=\left(\gamma \frac{w}{w_{\mu}}+(1-\gamma)\right) \delta^{u} \theta_{\mu}
$$

where $\gamma \in[0,1]$ denotes the degree of income redistribution of social insurance. Hence the previous section corresponds to the special case of maximum redistribution $\gamma=1$. For the minimum redistribution case $\gamma=0$ social insurance no longer redistributes between incomes. The latter case corresponds to an equivalence principle where expected insurance benefits are proportional to contributions. Our intention is to capture some feature of insurance against earnings loss which is much less redistributive even if contributions are proportional to income simply because the benefit is also proportional to income. We first study the case of voting over social insurance against private insurance before analysing the possibility to vote over the degree of private and social insurance mix.

\subsection{Social insurance versus private insurance}

Since private insurance is exclusive from social insurance, the degree of redistribution of social insurance does not affect the separating private insurance contracts, and thus the payoff function from private insurance is the same as in (4). On the other hand the payoff associated to full social insurance becomes,

$$
\begin{aligned}
V\left(\pi^{u}(w ; \gamma) ; \theta, w\right) & =w-\pi^{u}(w ; \gamma) \\
& =w-\left(\gamma \frac{w}{w_{\mu}}+(1-\gamma)\right) \theta_{\mu}
\end{aligned}
$$

Comparing (6) and (4) and fixing the degree of redistribution $\gamma \in[0,1]$, we can derive for each $\theta$ the marginal type $w=w_{\gamma}^{\circ}\left(\theta, \alpha, \theta_{\mu}, \bar{\theta}, w_{\mu}\right)$ who is indifferent between private and social insurance as the solution to

$$
\alpha \theta\left(1-(\theta / \bar{\theta})^{1 / \alpha}\right)+\theta=\gamma \frac{w}{w_{\mu}} \theta_{\mu}+(1-\gamma) \theta_{\mu} .
$$

It follows that

$$
\gamma \frac{w_{\gamma}^{\circ}(\theta ; \cdot)}{w_{\mu}}+(1-\gamma)=(1+\varphi(\alpha, \theta)) \frac{\theta}{\theta_{\mu}}
$$


where $\varphi(\alpha, \theta)=\alpha\left(1-(\theta / \bar{\theta})^{1 / \alpha}\right)>0$ is the same efficiency gain of social insurance as before. This generalises the indifference condition to various degrees of redistribution. In particular for $\gamma=1$ we recover condition (5). At the other extreme for $\gamma=0$, condition (7) is independent of income since in this case social insurance only redistributes across risks. This condition then suggests that even some individuals just below the mean risk may prefer social insurance due to efficiency gain (i.e., social insurance offers them better coverage relative to private insurance). Evidently all those agents with risk $\theta$ and income $w$ such that $w \leq w_{\gamma}^{\circ}(\theta ; \cdot)$ prefer social insurance over private insurance and vice versa. We verify that $w_{\gamma}^{\circ}(\theta ; \cdot)$ is increasing in $\theta$ almost everywhere and that it is above the mean income for any risk level above the average. Straightforward differentiation also gives that $w_{\gamma}^{\circ}(\theta ; \cdot)$ is increasing with risk aversion and mean income but is decreasing with average risk.

We then obtain the following proposition which extends proposition 1 to any degree of redistribution.

Proposition 4 There is a majority support for social insurance against private insurance if and only if

$$
\int_{\underline{\theta}}^{\bar{\theta}} \int_{\underline{w}}^{w_{\gamma}^{\circ}(\theta ; \cdot)} d H(\theta, w) \geq 1 / 2
$$

where $w_{\gamma}^{\circ}(\theta ; \cdot)$ is increasing in $\theta$ almost everywhere and risk aversion $(\alpha>0)$ implies that $w_{\gamma}^{\circ}(\theta ; \cdot)>w_{\mu}$ for all $\theta \geq \theta_{\mu}$. Moreover $w_{\gamma}^{\circ}(\theta ; \cdot)$ is increasing in $\alpha$ for each $\theta \in[\underline{\theta}, \bar{\theta})$ and so more risk aversion increases the political support for social insurance.

As for the effect of a change in $\gamma$ on the indifference condition we get that increasing $\gamma$ (more redistribution) induces a clockwise rotation of $w_{\gamma}^{\circ}(\theta ; \cdot)$ around $w=w_{\mu}$ in the $\left(\theta / \theta_{\mu}, w / w_{\mu}\right)$-space. The reason is that a larger $\gamma$ increases the importance of income in the preference for social insurance leading some low risk individuals below the mean income to switch their vote for social insurance but leading also some high risk individuals above the average income to switch to private insurance.

Table 4 shows the percentage of the population favouring social insurance for various $(\alpha, \gamma)$ when $w$ and $\theta$ are not correlated with symmetric distribution of risk and positively skewed distribution of income. It reveals that there is always a strict majority in favour of social insurance except 
when $\alpha=\gamma=0$ (i.e., no efficiency and income redistribution effect). Moreover the size of this majority is increasing with risk aversion $(\alpha)$ but rather unaffected by the degree of redistribution of social insurance $(\gamma)$. This is true even though the median income is less than the mean income.

Table 4: Social insurance support with uncorrelated risk and income

as a function of redistributiveness and risk aversion ${ }^{20}$
\begin{tabular}{|l|l|l|l|l|}
\hline & $\boldsymbol{\alpha}=\mathbf{0}$ & $\boldsymbol{\alpha}=\mathbf{0 . 1}$ & $\boldsymbol{\alpha}=\mathbf{0 . 2}$ & $\boldsymbol{\alpha}=\mathbf{1} / \mathbf{3}$ \\
\hline$\gamma=\mathbf{0}$ & $50.00 \%$ & $56.73 \%$ & $62.37 \%$ & $67.97 \%$ \\
\hline$\gamma=\mathbf{1} / \mathbf{4}$ & $50.03 \%$ & $56.68 \%$ & $62.22 \%$ & $67.75 \%$ \\
\hline$\gamma=\mathbf{1} / \mathbf{2}$ & $50.10 \%$ & $56.52 \%$ & $61.93 \%$ & $67.37 \%$ \\
\hline$\gamma=\mathbf{3 / 4}$ & $50.23 \%$ & $56.33 \%$ & $61.75 \%$ & $66.78 \%$ \\
\hline$\gamma=\mathbf{1}$ & $50.27 \%$ & $56.23 \%$ & $61.52 \%$ & $66.20 \%$ \\
\hline
\end{tabular}

We may wonder whether some correlation between income and risk could increase the sensitivity of the social insurance support to the degree of income redistribution. With positive correlation income redistribution and risk redistribution work in opposite directions: those with low incomes benefit from income redistribution but since they face low risks, they also subsidise higher risks. Therefore their preference for social insurance is weaker that in the case of negative correlation where both income and risk redistribution reinforce each other. We should then expect a change in the degree of income redistribution to have more effect with positive correlation than negative correlation. Table 5 shows that this is the case. Under negative correlation the support of social insurance is almost independent of the degree of redistribution. By contrast, when correlation is positive intensifying income redistribution increases significantly the social insurance support, especially when risk aversion is high.

Table 5: Social insurance support with correlated risk and income as a function of correlation, redistributiveness and risk aversion ${ }^{21}$

\footnotetext{
${ }^{20} w=3 x+1$ with $x \sim \operatorname{Beta}(2,3)$ so that $w \in[1,4]$

$\theta=2 y / 3$ with $y \sim \operatorname{Beta}(2,2)$ so that $\theta \in[0,2 / 3]$

${ }^{21} w=3 x+1$ with $x \sim \operatorname{Beta}(2,3)$ so that $w \in[1,4]$

$\theta=2 y / 3$ with $y \sim \operatorname{Beta}(2,2)$ so that $\theta \in[0,2 / 3]$
} 


\begin{tabular}{|l|l|l|l|l|l|}
\hline $\operatorname{corr}(w, \theta)$ & & $\boldsymbol{\alpha}=\mathbf{0}$ & $\boldsymbol{\alpha}=\mathbf{0 . 1}$ & $\boldsymbol{\alpha}=\mathbf{0 . 2}$ & $\boldsymbol{\alpha}=\mathbf{1} / \mathbf{3}$ \\
\hline \multirow{3}{*}{1} & $\gamma=\mathbf{0}$ & $50.00 \%$ & $56.73 \%$ & $62.37 \%$ & $67.97 \%$ \\
\cline { 2 - 6 } & $\gamma=\mathbf{1} / \mathbf{4}$ & $50.33 \%$ & $56.17 \%$ & $61.35 \%$ & $66.28 \%$ \\
\cline { 2 - 6 } & $\gamma=\mathbf{1} / \mathbf{2}$ & $50.55 \%$ & $55.85 \%$ & $60.57 \%$ & $64.97 \%$ \\
\cline { 2 - 6 } & $\gamma=\mathbf{3} / \mathbf{4}$ & $50.72 \%$ & $55.62 \%$ & $59.90 \%$ & $63.85 \%$ \\
\cline { 2 - 6 } & $\gamma=\mathbf{1}$ & $50.90 \%$ & $55.33 \%$ & $59.30 \%$ & $62.97 \%$ \\
\hline \hline \multirow{3}{*}{1} & $\gamma=\mathbf{0}$ & $50.00 \%$ & $56.73 \%$ & $62.37 \%$ & $67.97 \%$ \\
\cline { 2 - 6 } & $\gamma=\mathbf{1} / \mathbf{4}$ & $50.38 \%$ & $58.18 \%$ & $64.18 \%$ & $70.37 \%$ \\
\cline { 2 - 6 } & $\gamma=\mathbf{1} / \mathbf{2}$ & $50.92 \%$ & $60.20 \%$ & $66.67 \%$ & $72.90 \%$ \\
\cline { 2 - 6 } & $\gamma=\mathbf{3 / 4}$ & $51.58 \%$ & $62.32 \%$ & $70.23 \%$ & $76.23 \%$ \\
\cline { 2 - 6 } & $\gamma=\mathbf{1}$ & $53.00 \%$ & $65.90 \%$ & $73.88 \%$ & $81.43 \%$ \\
\hline
\end{tabular}

\section{$5.2 \quad$ Impossibility of top-up}

We now show that the impossibility of mixed insurance extends to any degree of redistribution of social insurance. Following the same line of reasoning as in Section 4, and changing the social insurance premium to $\pi^{u}(w ; \gamma)=$ $\left(\gamma \frac{w}{w_{\mu}}+(1-\gamma)\right) \delta^{u} \theta_{\mu}$, we obtain the following payoff function for an individual with risk $\theta$ and income $w$ :

$$
\begin{aligned}
V\left(\delta^{u}, \pi^{u}(w ; \gamma), \delta(\theta), \pi(\theta) ; \theta, w\right)= & w-\left(\gamma \frac{w}{w_{\mu}}+1-\gamma\right) \delta^{u} \theta_{\mu}-\left(\delta(\theta)-\delta^{u}\right) \theta \\
& -(1+\alpha)(1-\delta(\theta)) \theta
\end{aligned}
$$

In any separating equilibrium, the following necessary local incentive compatibility condition must hold: for all $\theta$

$$
\begin{aligned}
{\left[\frac{\partial V\left(\delta^{u}, \pi^{u}(w ; \gamma), \delta(\hat{\theta}), \pi(\hat{\theta}) ; \theta, w\right)}{\partial \hat{\theta}}\right]_{\hat{\theta} \rightarrow \theta} } & =(1+\alpha) \delta^{\prime}(\theta) \theta-\delta^{\prime}(\theta) \theta-\left(\delta(\theta)-\delta^{u}\right) \\
& =\alpha \theta \delta^{\prime}(\theta)-\left(\delta(\theta)-\delta^{u}\right)=0
\end{aligned}
$$

This expression is the same as the one obtained in Section 4 and so the set of separating private insurance contracts is unaffected by the degree of redistribution of social insurance. Changing $\gamma$ has no effect on the private insurance market. Using this separability property, we can plug the equilibrium private insurance contract derived in Section 4 into the payoff functions to get,

$$
V\left(\delta^{u}, \pi^{u}(w ; \gamma), \delta^{*}(\theta), \pi^{*}(\theta) ; \theta, w\right)=w-\left(\gamma \frac{w}{w_{\mu}}+1-\gamma\right) \delta^{u} \theta_{\mu}-\left(1-\delta^{u}\right)(\theta / \bar{\theta})^{1 / \alpha} \theta
$$




$$
-(1+\alpha)\left[1-\left(1-\delta^{u}\right)(\theta / \bar{\theta})^{1 / \alpha}-\delta^{u}\right] \theta
$$

Differentiating with respect to $\delta^{u} \in[0,1]$ we obtain,

$$
\frac{\partial V(\cdot)}{\partial \delta^{u}}=\theta-\left(\gamma \frac{w}{w_{\mu}}+1-\gamma\right) \theta_{\mu}+\alpha \theta\left[1-(\theta / \bar{\theta})^{1 / \alpha}\right]
$$

Since this expression is independent of $\delta^{u}$, for any degree of redistribution $\gamma \in[0,1]$ individuals have corner solution in terms of their preference for $\delta^{u}$, with a majority in favour of either no social insurance or full social insurance. Furthermore, the set of individuals preferring full social insurance is the same as when social and private insurance are mutually exclusive options, as can be seen by comparing (8) and (7). The next proposition follows immediately,

Proposition 5 Suppose that agents can supplement social insurance with private insurance and that they vote on the degree of social insurance coverage. Then for any joint distribution of risk and income in the population and regardless of the degree of redistribution of social insurance, under the dual theory of risk a majority favours either full social insurance or no social insurance at all. Mixing private and redistributive social insurance is not politically sustainable unless complete public coverage is precluded for some incentive reasons.

\section{The importance of adverse selection}

Adverse selection is clearly a key element in the political support for social insurance. One implication of adverse selection is that voluntary insurance can only offer incomplete coverage, which provides an efficiency case for compulsory social insurance. We believe it is thus important to understand the political feasibility of compulsory social insurance in the face of the growing possibility for (private) insurers to detect individual risks through genetic tests. ${ }^{22}$ To tackle this issue, we take the extreme view that it is possible to observe individual risks and that the private market will offer insurance contracts accordingly. Following the argument of the previous section, we assume a compulsory social insurance of the form $\left\{\delta^{u}, \pi^{u}(w ; \gamma)\right\}$ that can be supplemented by a voluntary private insurance at an actuarially fair price. Then, any risk averse individual with income $w$ and risk $\theta$ chooses full coverage $\delta(\theta)=1$, with the resulting payoff:

\footnotetext{
${ }^{22}$ See The Economist (19 October 2000) "Insurance in the genetic age", p23.
} 
$V^{*}\left(\delta^{u}, \pi^{u}(w ; \gamma), \delta^{*}(\theta), \pi^{*}(\theta) ; \theta, w\right)=w-\left(\gamma \frac{w}{w_{\mu}}+1-\gamma\right) \delta^{u} \theta_{\mu}-\left(1-\delta^{u}\right) \theta$

Differentiating this expression with respect to $\delta^{u} \in[0,1]$ we obtain

$$
\frac{\partial V^{*}(\cdot)}{\partial \delta^{u}}=\theta-\left(\gamma \frac{w}{w_{\mu}}+1-\gamma\right) \theta_{\mu}
$$

Since (9) is independent of $\delta^{u}$ we still obtain that the majority favours either a full social insurance or no social insurance at all. In other words, the top-up system is also politically unfeasible with perfect private insurance. Moreover the size of the group favouring social insurance decreases with perfect private insurance in presence of risk aversion. This is easily seen by comparing (8) and (9). In fact, the set of individuals favouring social insurance is

$$
\left\{(\theta, w): \theta \geq\left[\gamma \frac{w}{w_{\mu}}+(1-\gamma)\right] \theta_{\mu}\right\}
$$

representing a percentage of the population equal to

$$
\int_{\underline{w}}^{\bar{w}} \int_{\left[\gamma \frac{w}{w_{\mu}}+(1-\gamma)\right] \theta_{\mu}}^{\bar{\theta}} d H(\theta, w)
$$

Notice that the set of those favouring social insurance is now independent of risk aversion. This is because the existence of perfect private insurance enables individuals to get full coverage on the private market. One can ask whether, for correlated distributions of risk and income, a change in the redistribution level of social insurance could shift the majority away from social insurance. The answer is negative with a symmetrical risk distribution and an income distribution skewed to the right. This is readily seen from Table 5 setting $\alpha=0$ to neutralise for the adverse selection effect.

We can summarise our results on the political support for social insurance in the absence of adverse selection in the following proposition.

Proposition 6 Suppose that there is no adverse selection. Agents can supplement social insurance with perfect private insurance and vote on the 
degree of social insurance coverage. Then, mixing the two systems is never politically feasible under the dual theory of risk, regardless of the joint distribution of risk and income and of the degree of redistribution of social insurance. Moreover, the political support for social insurance is lower than in the presence of adverse selection, and this support is independent of risk aversion.

\section{Conclusion}

Most social insurance systems in the European Union and elsewhere include a significant amount of income redistribution in addition to the redistribution across risk levels. This paper has addressed the important question of the political support for social insurance in the presence of private alternatives. The efficiency argument for social insurance is that, by making insurance compulsory, it can overcome the adverse selection problems that plague the private insurance market. Combining this efficiency argument with the income redistribution effect of social insurance (due to income related contributions) and the risk redistribution effect (due to the pooling of risks) we have shown that, for a large class of joint distributions of risk and income in the population, there is a majority support for social insurance. In addition, higher risk aversion implies more support for social insurance, simply because adverse selection prevents private insurance from offering full coverage. However, if we remove the adverse selection problem (say, because genetic tests can make individual risks known to the private insurance companies) then the public support for social insurance will shrink and the majority in favour of social insurance will become more fragile. We have also shown that the so-called "top-up system" where individuals can voluntarily supplement social insurance with some private insurance is not politically sustainable in the sense that there is always a majority that would prefer either full or no social insurance at all. This result is mostly a consequence of our non-expected utility model but it suggests the more general point that the political demand for mixed insurance depends heavily on the modelling of risk aversion.

We should emphasise some limits of our analysis. The first is that we have assumed away any moral hazard considerations. Typically, the moral hazard problem is important in the insurance context and takes two different dimensions: underprevention (insurance coverage reduces prevention efforts) and overconsumption (insurance coverage inflates costs). In both cases, moral hazard calls for the use of deductible and co-insurance. However 
public (compulsory) insurance and private (voluntary) insurance are equally affected by this moral hazard problem, and so ignoring this aspect should not really affect the choice between the two systems.

A more important criticism of our analysis concerns the benefits that insurance provides. In this paper, we have assumed that all individuals face the same potential damage (as in Rothschild and Stiglitz, 1976). While this may seem a good approximation of health care systems, social insurance also provides (sometimes jointly with the employers) compensation for earnings loss. Introducing this aspect into the analysis will bring social insurance closer to the equivalence principle according to which benefits are proportional to contributions (both related to earnings). Since the consequence of allowing insurance against earnings loss is to decrease the redistributive effect of social insurance, then our results about the effect on the political support for social insurance of changing its redistributive content are relevant.

Acknowledgments. This paper has been prepared while Jean Hindriks was visiting CORE and Philippe De Donder was visiting the W. Allen Wallis Institute of Political Economy at the University of Rochester. Financial support from both institutions is gratefully acknowledged. We thank the Editor Steve Coate and two anonymous referees for helpful suggestions. Special thank goes to Jacques Dreze who wrote detailed comments leading to substantial improvements in the paper. We also would like to thank Dan Anderberg, Larry Epstein, Pierre Pestieau, Jean-Charles Rochet and seminar participants at Rochester, Université Laval, Université de Montreal, University of Illinois at Urbana Champaign, Yale University and at the Public Choice 2001 meeting in San Antonio for their comments and reactions on earlier versions of the paper.

\section{References}

[1] Anderberg, D., 1999, Determining the mix of public and private provision of insurance by majority rule, European Journal of Political Economy, 15, 417-40.

[2] Besley, T. and M. Gouveia, 1994, Alternative systems of health care provision, Economic Policy, 19, 199-258. 
[3] Blomqvist, A. and H. Horn, 1984, Public health insurance and optimal income taxation, Journal of Public Economics, 24, 352-71.

[4] Börsch-Supan, A. and M. Miegel (Eds.), 2001, Pension reform in six countries: What can we learn from each other?, Berlin: Springer.

[5] Casamatta G., Cremer, H. and P. Pestieau, 2000, Political sustainability and the design of social insurance, Journal of Public Economics, 75, 341-364.

[6] Cohen, M., 1995, Risk aversion concepts in expected and non-expected utility models, The Geneva Papers on Risk and Insurance Theory, 20, 73-91.

[7] Coronado, J.L., D. Fullerton and T. Glass, 2000, The progressivity of social security, NBER No 7520.

[8] Cremer, H. and P. Pestieau, 1996, Redistributive taxation and social insurance, International Tax and Public Finance, 3, 281-95.

[9] Doherty, N. and L. Eeckhoudt, 1995, Optimal insurance without expected utility: the dual theory and the linearity of insurance contracts, Journal of Risk and Uncertainty 10, 157-179.

[10] Eckstein, Z., M. Eichenbaum and D. Peled, 1985, Uncertain lifetimes and the welfare enhancing properties of annuity market and social security, Journal of Public Economics, 26, 303-26.

[11] Engers, M. and L. Fernandez, 1987, On the existence and uniqueness of signalling equilibria, Econometrica, 55, 425-40.

[12] Epple,D. and R.E. Romano, 1996, Public provision of private goods, Journal of Political Economy, 104, 57-84.

[13] Gouveia, M., 1997, Majority rule and the public provision of a private good, Public Choice, 93, 221-44.

[14] Guesnerie, R., 1995, A Contribution to the Pure Theory of Taxation, Cambridge: Cambridge University Press.

[15] Machina, M., 1995, Non-expected utility and the robustness of the classical insurance paradigm, in Gollier C. and M. Machina (eds.), Non Expected Utility and Risk Management, Boston: Kluwer Academic Press. 
[16] Mailath, G., 1987, Incentive compatibility in signaling games with a continuum of types, Econometrica, 55, 1349-65.

[17] Mossin, J., 1968, Aspects of rational insurance purchasing, Journal of Political Economy, 76, 533-68.

[18] Pauly, M., 1992, The normative and positive economics of minimum health benefits, in P. Zweifel and H. Frech (eds.): Health Economics Worldwide, Boston: Kluwer Academic Press.

[19] Peterson, N., 1986, A public choice analysis of parallel public-private provision of health care, in A. Culyer and B. Jonsson (eds.): Public and Private Health Services, Oxford, Basil Blackwell.

[20] Petretto, A., 1999, Optimal social health insurance with supplementary private insurance, Journal of Health Economics, 18, 727-45.

[21] Riley, J., 1979, Informational equilibrium, Econometrica, 47, 331-59.

[22] Riley, J., 1985, Competition with hidden knowledge, Journal of Political Economy, 93, 958-76.

[23] Rochet, J.-C, 1991, Incentives, redistribution, and social insurance, The Geneva Papers on Risk and Insurance Theory, 16, 143-65.

[24] Rothschild, M. and J.E. Stiglitz, 1976, Equilibrium in competitive insurance markets: an essay in the economics of incomplete information, Quarterly Journal of Economics, 90, 624-49.

[25] Segal, U. and A. Spivak, 1990, First order versus second order risk aversion, Journal of Economic Theory, 51, 111-125.

[26] Usher, D., 1977, The welfare economics of the socialization of commodities, Journal of Public Economics.

[27] Wagstaff, A. and E. van Doorslaer, 1999, Equity and the financing of health care: some further international comparisons, Journal of Health Economics, 18, 263-90.

[28] Yaari, M., 1986, Univariate and multivariate comparisons of risk aversion: a new approach, in Essays in Honor of Kenneth Arrow, ed. by W.P. Heller, R.Starr, and D. Starrett. Cambridge:Cambridge University Press. 
[29] Yaari, M., 1987, The dual theory of choice under risk, Econometrica 55 , N01, 95-115. 
Figure 1 : Critical value of $w^{\circ}$ Ew as a function of theta Etheta and of risk aversion

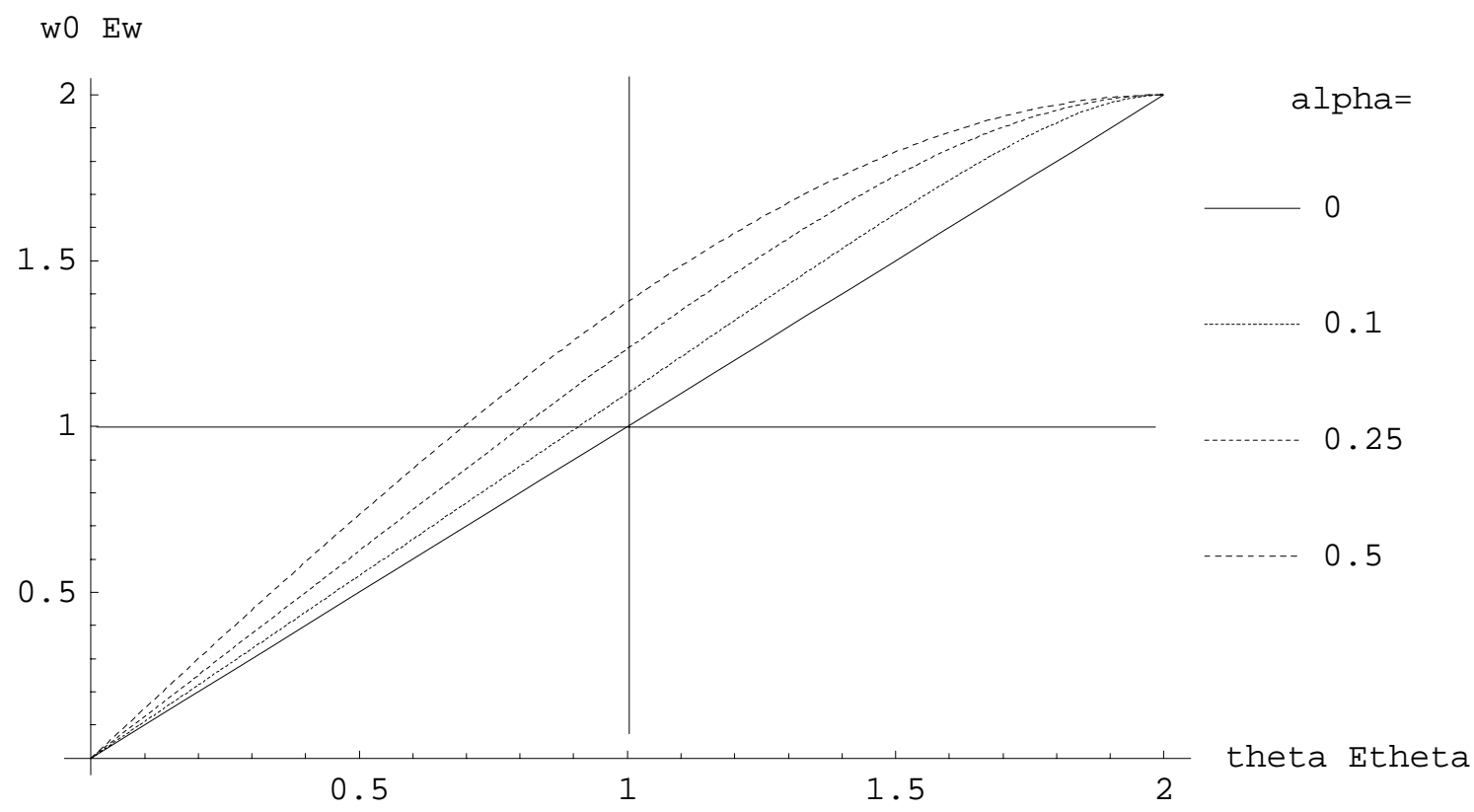

